\title{
40 km long-range Raman-based distributed temperature sensor with meter-scale spatial resolution
}

\author{
A. Signorini ${ }^{1}$, S. Faralli ${ }^{1}$, M.A. Soto ${ }^{1}$, G. Sacchi ${ }^{2}$, F. Baronti ${ }^{3}$, R. Barsacchi ${ }^{3}$, A. Lazzeri ${ }^{3}$, R. Roncella ${ }^{3}$, \\ G. Bolognini ${ }^{1}$, F. Di Pasquale ${ }^{1}$, \\ 1: Scuola Superiore Sant'Anna, Via G. Moruzzi 1,, 56124 Pisa, Italy.E-mail: al.signorini@sssup.it \\ 2: Fibersens S.r.l., viale Italia 10/A, Corsico (MI), Italy \\ 3: Dipartimento di Ingegneria dell'Informazione, Università di Pisa, Via Caruso 16, 56100 Pisa, Italy
}

\begin{abstract}
We propose a new Raman based distributed measurement technique which allows for temperature sensing over nearly $40 \mathrm{~km}$ of graded index multimode optical fiber with meter-scale spatial resolution and temperature accuracy better than $3{ }^{\circ} \mathrm{C}$.

(C) 2010 Optical Society of America

OCIS codes: (060. 2370) Fiber optics sensors, (190.5650) Scattering, Raman.
\end{abstract}

\section{Introduction}

Raman-based distributed temperature sensors (DTS) have been studied and have found successful implementation in many different application areas, from fire- and leakage-detection systems to power cable monitoring and security, where they offer unparallel sensing ability over thousands measuring points [1].

Almost all the distributed Raman sensing techniques described in literature, as well as those implemented in commercial systems, are based on optical time-domain reflectometry (OTDR), in which the temperature estimation is usually performed by measuring the Stokes (S) and anti-Stokes (AS) spontaneous Raman backscattering intensities generated by optical pulses propagating along the sensing fiber [2].

The main limitations in such Raman-based DTS systems arise essentially from the low backscattered Raman anti-Stokes power and consequent poor signal-to-noise ratio (SNR), limiting the maximum attainable sensing distance (actually Raman anti-Stokes intensity typically lies in the order of $\mathrm{nW}$-scale). An improvement in sensing performance by increasing the optical pulse power launched into the sensing fiber is only possible up to a given extent, due to the onset of optical non-linearities, mainly limited by stimulated Raman scattering (SRS) when using meter-scale spatial resolution. SRS actually induces distortion in the Raman backscattering traces whenever the optical power of light pulses exceeds a given threshold value. Although advanced techniques based on pulse coding [3] have been recently proposed in order to increase the available peak power levels in Raman-based DTS, without inducing fiber nonlinear effects, their practical development can be sometimes hindered by the technology underlying high-power pulsed lasers (e.g. Q-switched type) that are used in some schemes for long-range sensing systems with high spatial resolution [4], not allowing an easy implementation of pulse code-words.

In this paper, we propose, for the first time to our knowledge, a novel measurement technique which overcomes detrimental effects linked to fiber non-linearities impairments arising near the fiber input, thus greatly enhancing the sensing performance at the far fiber-end. By comparing with standard measuring techniques and considering the same total measurement time, we experimentally show that a significant enhanced accuracy can be achieved at the far fiber-end at the expense of a slightly worse behavior near the fiber input. Thus, this technique results in a more homogeneous accuracy throughout the sensing fiber compared to the strongly asymmetrical classical behavior of the sensor accuracy versus fiber distance. Unprecedented temperature sensing capabilities at nearly $40-\mathrm{km}$ distance are demonstrated with meter-scale spatial resolutions.

\section{Theory and measurement principle}

In Raman DTS, temperature estimation is commonly based on the measurement of backscattered anti-Stokes (AS) and Stokes (S) light intensities, $I_{A S}$ and $I_{S}$, which depend on the fiber temperature at the scattering point with the well-known relation $I_{A S} / I_{S} \propto \exp (h \Delta v / k T)$, where $h$ is the Planck constant, $k$ is the Boltzmann constant,

$T$ is the absolute temperature, and $\Delta v$ is the separation between Raman anti-Stokes and Stokes light frequencies. Whenever the pulsed pump power exceeds a given peak threshold value, some nonlinearities may take place, causing a distortion in the non-linear region of the AS and S traces. Considering 10-ns optical pulses, the maximum pulse power is actually limited by the SRS threshold, which is of the order of several watts in gradedindex multimode (MM) fibers. In fact, when using an input pulse power well above the SRS threshold, we observe that during propagation along the fiber the pump peak power decreases due to fiber loss and induced pump depletion, and eventually falls below the nonlinear threshold level after a given propagation distance. Therefore, optical linear regime is again observed in the far sensing fiber-end; in such a condition we have generation of spontaneous AS and S lights, which can then be used for a successful temperature estimation in the far fiber-end region with a higher accuracy with respect to a fully linear measurement (due to high pump 


\section{OWL2.pdf}

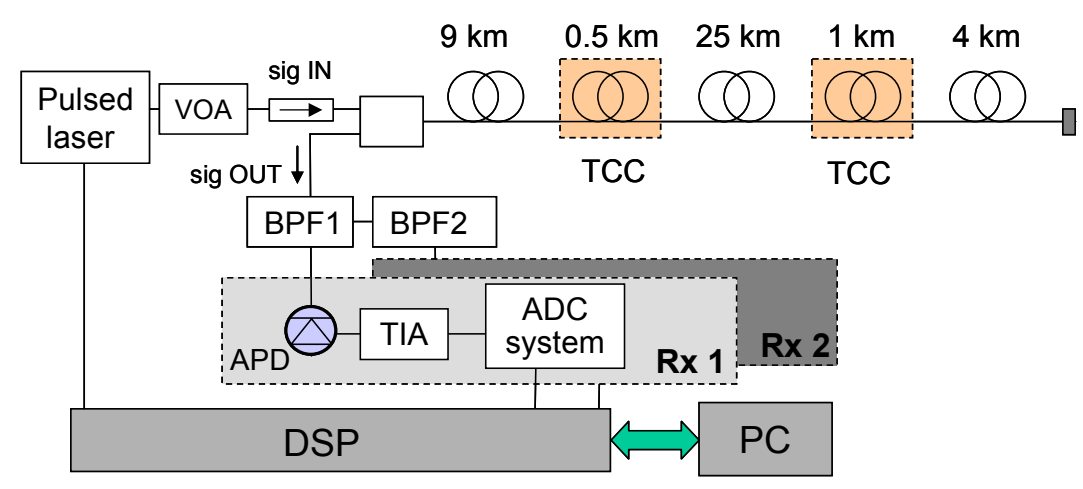

Fig. 1. Experimental set-up

power level attained at long fiber distances). The temperature estimation near the fiber input, where non-linear effects are occurring, can be carried out by performing another measurement session (which can precede the measurement session in nonlinear regime) through acquisition of traces employing lower pump peak power.

As a general measurement principle, the acquisition needs to be performed in two steps: in the first step (linear measurement session, low pump power) the temperature profile is obtained along the first half of the sensing fiber only (accuracy is not acceptable at long distances due to low pump peak power); in the second measurement session (non-linear measurement session, high pump power) the temperature profile is obtained in the second half of the fiber only (actually the acquired traces are now distorted near fiber input but we do not consider them for temperature evaluation). The two temperature profiles are then joined together to provide a high-accuracy, long-distance temperature measurement along the whole optical fiber. However, several effects, which can have an impact on the measurement accuracy and reliability, must be taken into account. Most notably, trace distortion in the second half of the sensing fiber originating from nonlinear effects in the first fiber part, can have a non-negligible impact in the estimated temperature. This can be however taken into account by a suitable trace normalization carried out by using the temperature and trace information obtained during the linear measurement session, allowing for a reliable and accurate temperature estimation also in the far fiber end region.

\section{Experimental set-up}

The schematic diagram of the implemented experimental setup for Raman-based DTS is shown in Fig 1 and is based on modifications of a long-range commercial DTS system (courtesy of Fibersens S.r.1.). The pulsed pump light is provided by a pulsed laser operating at $1550 \mathrm{~nm}$ with an average output power $\mathrm{P}_{A V}=1.5 \mathrm{~mW}$. The laser output power is controlled by a variable optical attenuator (VOA) to perform different measurement sessions for the first and second fiber parts. The pulse width of the laser is $10 \mathrm{~ns}$ with a repetition rate of $2.5 \mathrm{kHz}$. The backscattered Raman light from the sensing fiber is separated into the Stokes and anti-Stokes light components by a wavelength selective optical filter cascade. Two high-sensitivity low-noise avalanche photodiodes (APD) allow for simultaneous measurement of anti-Stokes and Stokes backscattered lights. Each APD is followed by a transimpedance amplifier (TIA) and proper amplification stages. Thus, then the output signals are sampled using a customized high-resolution analog-to-digital conversion (ADC) system. The sensing fiber consists of $39.2 \mathrm{~km}$ of graded-index multimode fiber (50/125 $\mu \mathrm{m}$ core/cladding diameters). Two sections of the sensing fiber with a length of $500 \mathrm{~m}$ and $1 \mathrm{~km}$ (at $\sim 9-\mathrm{km}$ and $\sim 35-\mathrm{km}$ distance respectively) are inserted in a temperature-controlled chamber (TCC), allowing for an accurate sensor characterization.

\section{Results}

Temperature measurements have been carried out for a set of different conditions considering both linear and nonlinear operating regimes. Note that due to different wavelength dependent losses and Raman scattering coefficients for different fiber spools, a pre-calibration procedure is required by measuring the AS to S intensity ratio for each fiber spool.

We have in particular investigated the temperature resolution, quantified as the root mean square (rms) noise in the measured temperature distribution, in both linear and nonlinear conditions for respective fiber portion.

Fig. 2 shows the anti-Stokes traces measured along the second half of the sensing fiber $(20-40 \mathrm{~km})$, with $1 \mathrm{~km}$ of fiber, at 36-km distance, inside the TCC set at different temperatures. These traces have been acquired in nonlinear condition by launching pulses at the fiber input with peak power well above the nonlinear threshold level, which is approximately $\sim 20 \mathrm{~W}$ when using 10-ns pulses. We can clearly see the typical exponential decay of the AS intensity in the second half of the fiber and note that the different temperatures are well distinguished near the far fiber-end. The same measurement in linear regime does not allow to accurately measure the temperature profile at the fiber end, due to high noise in the measurement and consequent poorer SNR. 


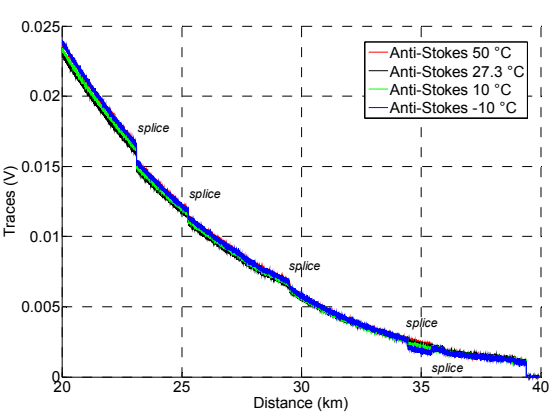

Fig. 2. Anti-Stokes traces in nonlinear regime

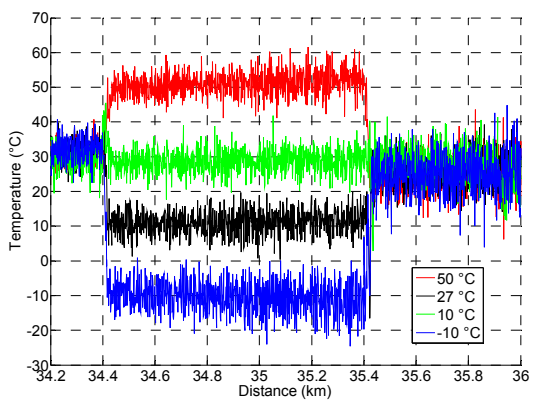

Fig. 3. Temperature distribution at the far fiber end

Fig. 3 shows a zoom of the temperature distribution near the far fiber-end (from $34.2 \mathrm{~km}$ to $36 \mathrm{~km}$ ), well confirming that the proposed measurement technique allows for a high accuracy over a very long measurement range, with an unprecedented performance over such a long range. Note that the total measurement time is less than 5 minutes, including two successive measurement sessions, one in linear condition to characterize the first half of the sensing fiber, and one in nonlinear condition for the second half, not considering the distorted S and AS traces acquired near the fiber input.

The attained temperature resolution is shown in Fig. 4, reporting rms resolution versus distance for the full measurement stage including both linear $(0-20 \mathrm{~km})$ and non-linear $(20-40 \mathrm{~km})$ measurement sessions. We can clearly see that the new technique allows for a rms temperature resolution reduced down to $3^{\circ} \mathrm{C}$, while a standard measurement does not provide acceptable resolution at the fiber end, as also shown in Fig. 4 (the

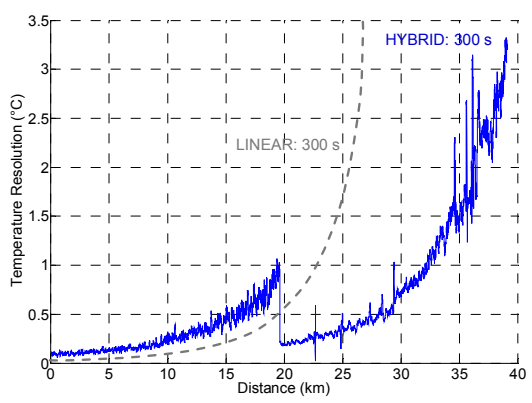

Fig. 4. Temperature resolution

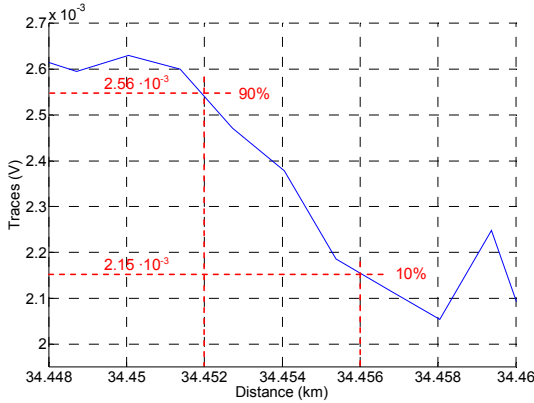

Fig. 5. Spatial resolution at $34.5 \mathrm{~km}$

temperature resolution with a single linear measurement session and same measurement time is worse than $25^{\circ} \mathrm{C}$ at the fiber end). Also note that the discontinuity in Fig. 4 is inherent with the proposed measurement technique which divides the measurement range into two sections. By performing a single measurement section all along the 40 -km fiber we would achieve a slightly better resolution at $20 \mathrm{~km}\left(\sim 0.7^{\circ} \mathrm{C}\right.$ instead of $\left.\sim 1{ }^{\circ} \mathrm{C}\right)$ but a much worst performance at the fiber end $\left(25^{\circ} \mathrm{C}\right.$ instead of $\sim 3^{\circ} \mathrm{C}$, not shown in Fig. 4).

Also the spatial resolution along the sensing fiber can be computed, by observing the measured temperature profile versus distance corresponding to a near step-like temperature variation, such at the input of the TCC. Note that although the pulse duration of $10 \mathrm{~ns}$ at the fiber input could in principle provide $1 \mathrm{~m}$ spatial resolution, intermodal dispersion broadens the pulse during propagation along the multimode fiber, degrading the spatial resolution; in our measured we observed a spatial resolution of about $1.2 \mathrm{~m}$ at 10-km distance and $\sim 4 \mathrm{~m}$ near the fiber end (this can be seen from Fig. 5 showing a 10\%-90\% step-response measurement around 34-km).

In conclusion we have proposed and experimentally demonstrated a new Raman based distributed measurement technique which allows for temperature sensing over nearly $40-\mathrm{km}$ graded index multimode optical fiber, with a promising application e.g. to leakage detection in gas/oil pipelines and in power cable monitoring. This novel technique, employing a hybrid scheme with two different peak power values and operation in non-linear regime, can achieve about $9 \mathrm{~dB}$ improvement with respect to standard technique, attaining a temperature accuracy better than $3^{\circ} \mathrm{C}$ with an acquisition time shorter than 5 min and a meter-scale spatial resolution. DTS systems based on this technique can be proved very effective for practical applications such gas/oil pipelines and power cables monitoring.

\section{References}

[1] Nature Photonics, “Optical-fibre Sensors," Tech. Focus, vol. 2, pp. 143-158, 2008.

[2] B. Culshaw, "Optical fiber sensor technologies: opportunities and-perhaps-pitfalls," J. Lightwave Technol., vol. 22, no. 1, pp. $39-50,2004$.

[3] G. Bolognini et al., "Performance Enhancement of Raman-based Distributed Temperature Sensors using Simplex Codes", in OFC 2006 Tech. Dig., paper OTuL1.

[4] M. A. Farahani et al., "Spontaneous Raman scattering in optical fibers with modulated probe light for distributed temperature Raman remote sensing", J. Lightwave Technol., 17, 1379-1391, 1999. 